\title{
The papillary muscle sling for ischemic mitral regurgitation
}

\author{
Ulrik Hvass, MD, and Thomas Joudinaud, MD
}

Objective: Our objective was to evaluate long-term stability of mitral repair and reverse remodeling in patients with severe ischemic left ventricular dysfunction and functional mitral regurgitation.

\begin{abstract}
Methods: Since June 2000, a total of 37 patients with ischemic functional mitral regurgitation have benefited from a double-level mitral repair that comprises an intraventricular peripapillary muscle sling completed by a classic intra-atrial mitral annuloplasty ring (mean age, 56 years; left ventricular end-diastolic diameter, $70 \pm 0 \mathrm{~mm}$; left ventricular end-systolic diameter, $55 \pm 5.6 \mathrm{~mm}$; ejection fraction, $15 \%$ to $45 \%$; pulmonary hypertension $>$ 60 in all patients; all were in New York Heart Association class III-IV). All patients had both papillary muscles encircled with a 4-mm polytetrafluoroethylene tube, correcting their lateral and downward displacement. Annuloplasty rings were moderately undersized or normal. Efficiency was evaluated on mitral stability, ventricular parameters, and functional status. According to the Leyden algorithm based on preoperative end-diastolic and end-systolic left ventricular diameters, only a minority of our patients were expected to experience reverse remodeling.
\end{abstract}

Results: Regurgitation is none to trivial in 31 and mild to moderate in 4. Follow-up (3-84 months; mean, $55 \pm 22$ months) shows stability of all initially successful double-level mitral repairs. Follow-up beyond 1 year shows improvements in ventricular diameters $(56 \pm 5 \mathrm{~mm})$, ejection fraction $(49 \pm 6)$, volume $(130 \pm 10 \mathrm{~mL})$, and sphericity index (0.55). Two patients died during follow-up and 1 underwent transplantation.

Conclusion: Reapproximating the papillary muscles has an immediate effect on mitral leaflet mobility by suppressing the tethering resulting from displacement of the papillary muscles. It has an effect in preventing recurrent mitral regurgitation by avoiding further papillary muscle displacement. In this cohort of severely disabled patients, reverse remodeling can be expected with the double-level repair. (J Thorac Cardiovasc Surg 2010;139:418-23)

Abnormal displacement of the papillary muscles (PMs) leading to excessive tension on chordae ${ }^{1,2}$ has been demonstrated in patients with left ventricular (LV) dysfunction and functional mitral regurgitation (FMR). The tethering, restricting mitral leaflet mobility, is instrumental in producing mitral regurgitation (MR) with an otherwise normal mitral valve. Mitral repair should consider all the intervening components, suggesting that correction of FMR should also focus on some form of intraventricular intervention directed to rectify the PM displacement, relieving tension on chordae.

On the basis of these considerations, we tested a new approach that consists in correcting, via the left atrium and through the mitral orifice, the abnormal displacement of the PMs using an intraventricular sling (Gore-Tex [polytetrafluoroethylene] 4-mm tube; W. L. Gore \& Associates, Inc, Flagstaff, Ariz) encircling the trabecular base of both PMs. Tightening and securing this sling reapproximates

From the Department of Cardiovascular Surgery, Bichat Hospital, Paris, France. Disclosures: None.

Read at the Eighty-ninth Annual Meeting of The American Association for Thoracic Surgery, Boston, Mass, May 9-13, 2009.

Received for publication June 11, 2009; revisions received July 23, 2009; accepted for publication Aug 9, 2009.

Address for reprints: Hvass Ulrik, MD, Bichat Hospital, 46 rue Henri Huchard, Paris 75018, France (E-mail: ulrik.hvass@bch.ap-hop-paris.fr).

0022-5223/\$36.00

Copyright (c) 2010 by The American Association for Thoracic Surgery doi: $10.1016 /$ j.jtcvs.2009.08.007 the two PMs and sets a more normal alignment with the overhead mitral structures.

Re-establishing the annulus-PM relationship was expected to relieve the excess tethering on the mitral leaflets and significantly restore leaflet mobility. A moderately undersized mitral annuloplasty ring would then complete the repair. The last 10 patients received a normally sized prosthetic mitral ring.

The intended procedure would thus comprise a double mitral ring: one inside the ventricle at the base of the PMs and the other on the mitral annulus.

Although mitral annuloplasty alone , $^{3,4}$ already offers symptomatic improvement in patients with FMR, occasionally even seriously undersizing a mitral ring does not give the anticipated result in terms of correction of MR. Reported recurrence rates of MR after ring annuloplasty alone are far from negligible. ${ }^{5,6}$ The initial intention and the expected advantages of this new technique were to make mitral repair more logical by addressing both components of the FMR (the displaced PMs and the annular deformities). We also expected the procedure to lower the incidence of recurrent MR resulting from continuing LV dilation and further displacement of the PMs liable to increase tension on the mitral chordae. An effect on regional wall motion was also theorized, related to the posterior folding of the left ventricle between the two PMs, ${ }^{7,8}$ affecting the ratio between volume and mass. After having initially reported results in $2003,{ }^{9}$ we can now give the medium-term results of this technique, results that lose 


\section{Abbreviations and Acronyms \\ FMR = functional mitral regurgitation \\ $\mathrm{LV} \quad=$ left ventricular \\ $\mathrm{MR}=$ mitral regurgitation \\ NYHA $=$ New York Heart Association \\ PMs = papillary muscles}

significance beyond 5 years owing to the small number of patients.

\section{PATIENTS AND METHODS \\ Patients}

FMR was diagnosed as ischemic in patients with coronary artery disease having had inferior and/or lateral myocardial infarctions. The mitral leaflets were normal, with restricted motion and no other available cause for MR. Diagnosis was discussed by cardiologists and accepted as such before the patients were enrolled for the operation. Since June 2000, a total of 37 patients (Table 1) with FMR related to ischemia have benefited from a double repair associating an intraventricular peripapillary muscle sling completed by a classic intra-atrial mitral annuloplasty ring. Ages ranged from 29 to 78 years, mean 56 years. Ejection fraction on angiograms was between $15 \%$ and $45 \%$. LV end-diastolic and end-systolic diameters were respectively $70 \pm 0 \mathrm{~mm}$ and $55 \pm 5.6 \mathrm{~mm}$. All patients had a systolic pulmonary arterial pressure above $60 \mathrm{~mm} \mathrm{Hg}$. MR was evaluated as moderate to moderately severe, or 2 to 4 on a scale of 4 . Medical therapy was maximal and all the patients had had several hospital admissions for LV failure. All patients were in New York Heart Association (NYHA) class III-IV.

\section{Surgical Technique}

Once the mitral valve had been exposed and analyzed as anatomically normal, we placed the stitches on the mitral annulus preparing for the annuloplasty. Slight traction on these sutures allows a better exposure of the subvalvular apparatus. The position of the anterior PM usually seems normal, under the anterior mitral commissure. The posterior PM is usually out of the field and it may be necessary to place a textile under the heart to visualize it correctly and gain adequate access. We start by working a blunt dissector through the trabeculations at the base of the posterior PM, ensuring that the tube will not be able to later migrate toward the head of the PM. The tube is then drawn around the base of the anterior PM. The loop is progressively tightened until the two PMs are in close contact. The loop is not squeezed tight to avoid any ischemia. The 4-mm polytetrafluoroethylene tube forms an intraventricular ring that is secured with strong sutures. Once tightened, there is no residual gap between the bases of the two PMs. No sutures are placed on the PMs themselves. The procedure is completed by mitral annuloplasty. Annuloplasty rings are considered of normal size when they cover the surface of the anterior mitral leaflet. Additional surgery comprised coronary bypass grafts in all patients, tricuspid annuloplasty in 5, and aortic valve replacement in 1 patient.

\section{Echocardiographic Follow-up}

To assess whether this new technique translates into quantifiable criteria, we evaluated the changes in the tenting effect MR and LV parameters that comprised LV diameters measured in systole and diastole, volume, sphericity index, and ejection fraction. These changes are compared with the preoperative values in Table 1.

Tension on chordae resulting from apical and/or posterior displacement of the mitral PMs will translate into a tenting effect that can be quantified on 2-dimensional echocardiogram either by the area enclosed between the an-
TABLE 1. Echocardiographic results after repair for ischemic mitral regurgitation

\begin{tabular}{lcccc}
\hline & Preoperative & Six months & One year & Five years \\
\hline Age $(\mathrm{y})$ & $56(29-78)$ & & & \\
LVEDD $(\mathrm{mm})$ & $70 \pm 0$ & $62 \pm 3.5$ & $56 \pm 5.5$ & $55 \pm 3.5$ \\
LVESD $(\mathrm{mm})$ & $55 \pm 5.6$ & $51.5 \pm 4.9$ & $50 \pm 5.5$ & $51 \pm 5.5$ \\
EF $(\%)$ & $29.5 \pm 5.5$ & $44 \pm 13$ & $49 \pm 6$ & $45 \pm 5$ \\
LVV $(\mathrm{mL})$ & $250 \pm 25$ & $160 \pm 18$ & $130 \pm 10$ & $122 \pm 15$ \\
MR 1-2/3-4 & $7 / 30$ & $32 / 3$ & $32 / 3$ & $31 / 0$ \\
TM gradients & $4.8 \pm 2.6$ & $3.2 \pm 1.6$ & $3.5 \pm 1.2$ & $4.4 \pm 1$ \\
Tenting (mm) & $14 \pm 2.1$ & $4 \pm 1.1$ & $\mathrm{NA}$ & $\mathrm{NA}$ \\
IPMD (mm) & $3.6 \pm 1.2$ & $0,8 \pm 0,3$ & $\mathrm{NA}$ & $\mathrm{NA}$ \\
PAP (mm Hg) & $>60$ & $35 \pm 15$ & $45 \pm 11$ & $41 \pm 9$ \\
Sphericity & 0.78 & 0.68 & 0.55 & $\mathrm{NA}$ \\
S/P wall mm & $8 / 7$ & $10 / 8$ & $11 / 8$ & $13 / 9$ \\
NYHAI-II/III-IV & $0 / 37$ & $30 / 5$ & $31 / 4$ & $29 / 2$ \\
\hline
\end{tabular}

$L V E D D$, Left ventricular end-diastolic diameter; $L V E S D$, left ventricular end-systolic diameter; $E F$, ejection fraction; $L V V$, left ventricular volume; $M R$, mitral regurgitation grade 1 to 2 over grade 3 to $4 ; T M$ gradients, transmitral gradients; IPMD, interpapillary muscle distance; $P A P$, pulmonary artery pressure; $S / P$ wall, septal/posterior left ventricular wall thickness; $N Y H A$, New York Heart Association; NA, not available.

nular plane and mitral leaflets from parasternal long-axis view at early and late systole or by the vertical distance between leaflet coaptation and the mitral annulus plane. Using the vertical distance criteria, the preoperative tenting measured $14 \pm 2.1 \mathrm{~mm}$. The mitral tenting parallels the displacement of mitral coaptation toward the LV apex. Tenting is not attenuated by annuloplasty alone and therefore this feature may serve as an objective criterion to evaluate the immediate effect of the PM sling on mitral leaflet mobility.

Residual MR was evaluated by transesophageal echocardiography about 3 months postoperatively and usually by transthoracic echocardiography thereafter.

\section{RESULTS (TABLE 1) \\ Clinical Results}

Positioning the intraventricular sling by approaching the PMs through the mitral orifice was possible in all patients. Approximating the two PMs immediately unloaded tension on the mitral chordae, improving leaflet mobility and coaptation. Crossclamping time was initially prolonged a mean $28 \pm 3.5$ minutes. Time devoted to encircling the PMs is now around 5 minutes.

Mitral annular dilatation was corrected with a moderately undersized or normally sized ring, sizes $30 \pm 2.8 \mathrm{~mm}$. In preference to the flexible rings we initially used in these patients, we switched to the Carpentier Physio ring (Baxter Healthcare Corp, Irvine, Calif) to compensate for the lack of mitral annular contractility related to ischemic mitral dysfunction.

Coronary bypass grafts comprise $2 \pm 1.15$ grafts per patient. The left internal thoracic artery was always used for the left anterior descending coronary artery. Bilateral thoracic arteries were not used in any of these patients. Three patients with coronary stents did not require surgical coronary artery bypass. 


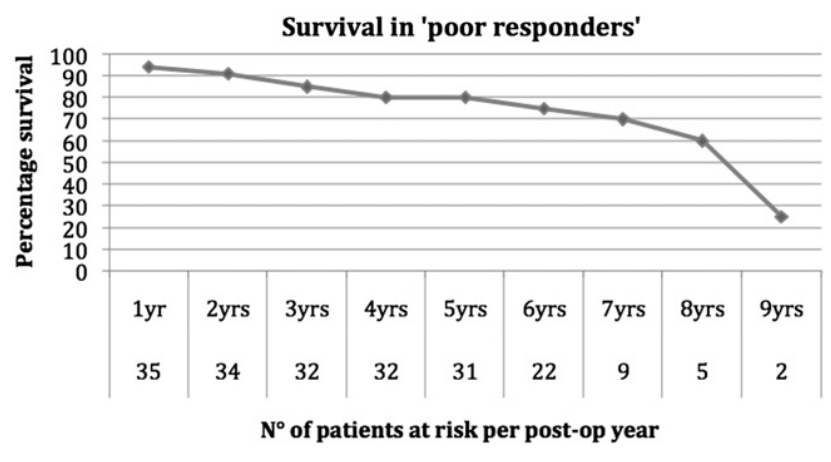

FIGURE 1. Survival in "poor responders."

Thirty-five patients survived the postoperative period (Figure 1). The 2 patients who died had a preoperative ejection fraction of $15 \%$, and 1 also had a calcified aortic valve. Intra-aortic balloon pumping was necessary in 1 patient preoperatively and in 5 other patients after cardiopulmonary bypass. Two pacemakers were inserted during the first postoperative month. During the mean follow-up of $55 \pm 22$ months (range, 3-84 months), the patients were regularly seen by the referring cardiologist. Two patients were reoperated on during the third year: 1 had a mitral valve replacement and the other unsuccessfully underwent transplantation. One patient died of noncardiac disease: a gangrenous cholecystitis. At 5 years, $80 \%$ were alive. Two of these patients, although in significantly improved condition and free from congestive heart failure, continued to receive higher-dose medications with lower ejection fractions owing to multiple preoperative infarctions. Beyond 6 years, the number of patients at risk is too small to be informative. Two transient ischemic attacks occurred; no endocarditis or anticoagulant-related bleeding was recorded. Five patients were on a regimen of long-term anticoagulation.

\section{Echocardiographic Results}

Echocardiography was performed on a yearly basis in all patients, either by the referring cardiologist or in the hospital facilities. Early residual regurgitation was none to trivial in 31 and mild in 2 . Results have been stable with no recurrence or accentuation of MR and no echocardiographic change in the positioning of the polytetrafluoroethylene sling. In 2 patients residual MR was moderately severe; they underwent reoperation after 3 years. One patient had had a flexible mitral ring, and the early unsatisfactory result made us switch over to rigid rings in these patients to compensate for the loss of annular contractility. The second patient had heart transplantation, residual regurgitation having been explained by our having left out a significant group of posterior PMs that had not been incorporated, unintentionally, within the sling. The preoperative parameters in these 2 patients did not differentiate them from the entire group.

The tenting effect on the mitral leaflets described by Yiu and associates ${ }^{1}$ is considerably attenuated as shown by com- paring the preoperative and postoperative 2-dimensional echocardiograms (Figure 2) even though the PM displacements are probably not fully corrected, in particular the apical displacement. The tenting effect, measured as the distance between mitral annulus plane and leaflet coaptation, varies from a preoperative mean value of $14 \pm 2.1 \mathrm{~mm}$ to a postoperative mean value of $4 \pm 1.1 \mathrm{~mm}$. Patients with the longer follow-up show no secondary displacement of the intraventricular sling. The distance between the PMs changed from a preoperative value of $3.6 \pm 1.2 \mathrm{~mm}$ to 0.8 $\pm 0.3 \mathrm{~mm}$. Postoperative measurements were made at the level where the sling was detected (Figure 3). Postoperative
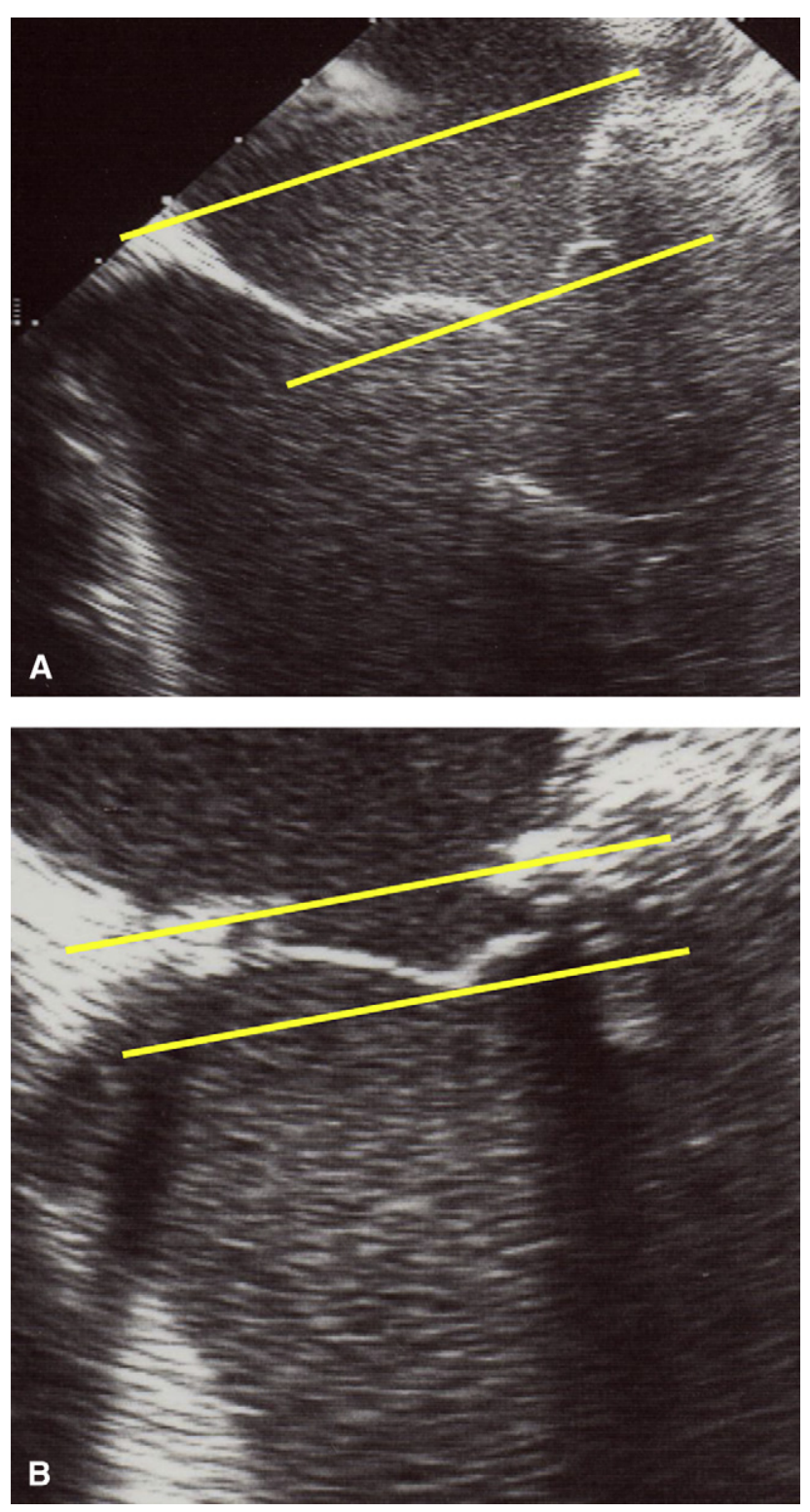

FIGURE 2. Preoperative (A) and postoperative (B) mitral valve, visualizing the change in mitral tenting (vertical height between the 2 lines) and coaptation point. 

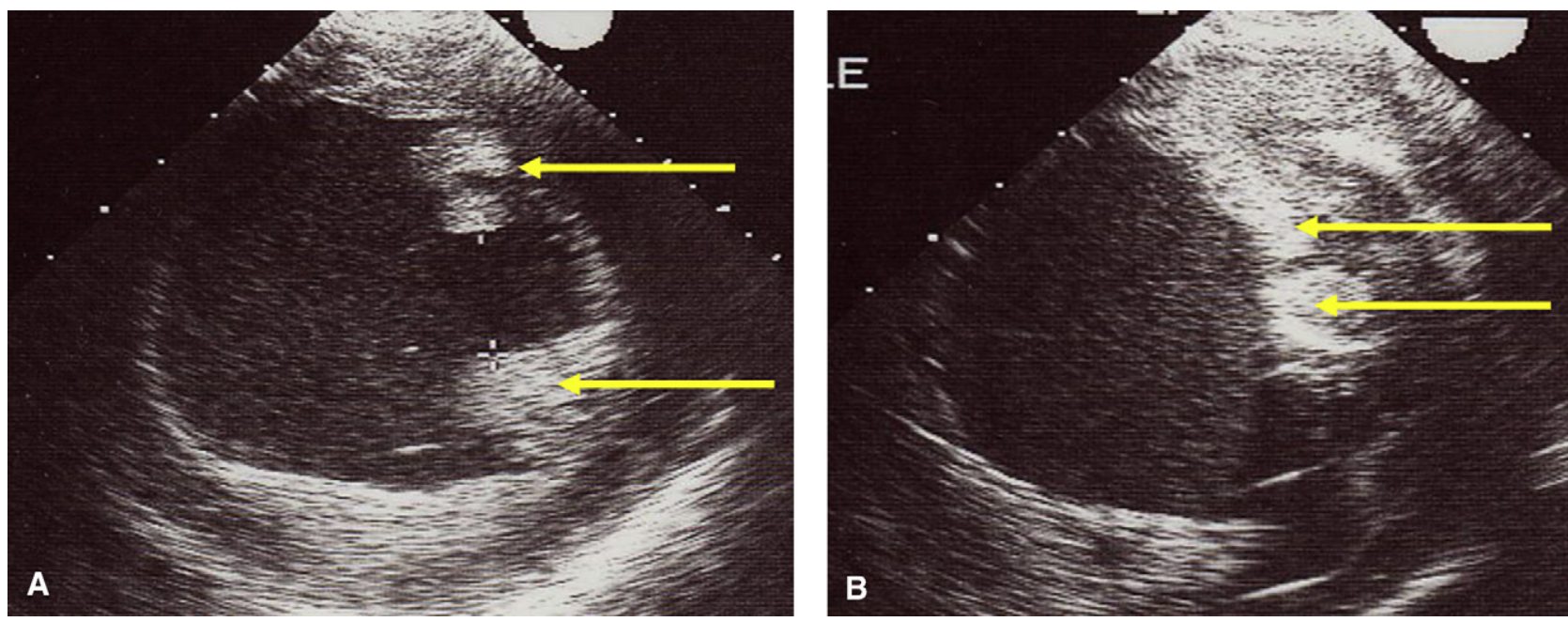

FIGURE 3. Short-axis transgastric echocardiogram showing the position of the papillary muscles (arrows) before (A) and after (B) placement of the intraventricular sling encircling both papillary muscles.

transvalvular mitral gradients ranged from 2 to $6 \mathrm{~mm} \mathrm{Hg}$, mean $3.2 \pm 1.6 \mathrm{~mm} \mathrm{Hg}$.

Preoperative and postoperative LV end-diastolic diameters diminished from $70 \pm 0 \mathrm{~mm}$ to $62.4 \pm 3.5 \mathrm{~mm}$ at 6 months and $56 \pm 5.5 \mathrm{~mm}$ at 1 year. End-systolic LV diameters changed respectively from $55 \pm 5.6 \mathrm{~mm}$ to $51.5 \pm 4.9$ $\mathrm{mm}$ and $50 \pm 5.5 \mathrm{~mm}$. Ejection fractions were, respectively, $29 \% \pm 5.5 \%, 44 \% \pm 13 \%$, and $49 \% \pm 6 \%$ at 1 year $(\mathrm{Ta}-$ ble 1). Pulmonary artery pressure lowered rapidly from greater than $60 \mathrm{~mm} \mathrm{Hg}$ to $35 \pm 15 \mathrm{~mm} \mathrm{Hg}$ at 6 months except in patients with significant residual MR who underwent reoperation.

At 6 months, functional status improved from NYHA IIIIV to NYHA I-II in all patients except the 2 who needed reinterventions.

\section{DISCUSSION}

In patients with ischemic FMR, local LV alterations mainly affect the posterior PM and the posterior and lateral LV wall. Chordal tethering subsequent to local remodeling intensifies MR that reflects the incomplete leaflet coaptation. A cascade of dysfunctional remodeling is initiated that comprises a mutual intricacy of further ventricular dilatation, augmented chordal tethering, MR, annular dilatation, and increased heart failure. Mortality in patients with dilated heart failure is related to the severity of LV systolic dysfunction, whereas FMR is a marker of poorer prognosis ${ }^{10}$ with a 1year mortality reported between $54 \%$ and $70 \%$.

Surgical management classically relies on significantly undersizing the mitral annuloplasty ring, decreasing valve area to increase leaflet apposition, and aiming to compensate for the reduced amplitude of mitral leaflet mobility as well as the loss of systolic annular contraction.
Such repairs reported by Bolling and associates, ${ }^{3}$ although taking into account only one of the components of FMR, claim 1- and 2-year survivals of $80 \%$ and $70 \%$ and demonstrate improvements for ejection fraction and cardiac output, NYHA class, and peak maximal volume of oxygen. These results are considered to reflect efficient remodeling with a reduction in sphericity index, regurgitant fraction, and end-diastolic volumes. Significantly undersizing the mitral annular remodeling ring overcorrects the coaptation zone and is thought to contribute to improvement seen in these myopathic hearts through acute remodeling of the base of the heart.

After more than a decade of enthusiasm generated by the pioneering works of Bolling and associates, ${ }^{3}$ discordant voices were heard claiming that MR recurs in $20 \%$ to $50 \%$ of the cases after only a few years. Recurrent MR after an initially successful mitral annuloplasty may be related to continuing PM displacement that augments the tethering on the leaflets, deranging once more the ratio between mitral orifice and covering surface of the mitral leaflets.

Recurrent MR seemed to be related to continuing or insufficient reversal of LV adverse remodeling. ${ }^{11,12}$ Thus two populations emerged according to the ventricular reaction: "good responders" and "poor responders," whom the Leyden algorithm separated according to the preoperative LV diameters. ${ }^{13,14}$ If an undersized mitral ring with a good coaptation height may be sufficient in good responders, how should one address the left ventricle in patients who were poor or boarderline responders?

Several new lines of investigation ${ }^{15-20}$ have been or are being evaluated, such as intraventricular patch remodeling and volume reduction of the left ventricle, plicating the posterior LV wall from the outside and bringing the PMs closer 
together. Transaortic resection of basal chordae of the anterior leaflet is also expected to relieve tension on the leaflet and enhance mobility of the mitral leaflets, but concern has been expressed regarding the role of these particular chordae in ventricular remodeling.

The papillary muscle sling has a double effect:

1. The sling addresses the mitral valve by proposing a repair that takes the mitral apparatus into account as a whole functioning unit. Furthermore, by suppressing the possibility of continual lateral papillary muscle displacement, the sling prevents recurrent mitral leaks. None of our patients with an initially perfect result, although the mean follow-up is only of 36 months, showed any secondary change compared with the initial result.

2. The sling also addresses the left ventricle. By this transmitral approach, plicating the posterior wall of the left ventricle from the inside, the sling acutely changes the shape and the volume of the left ventricle. Shortening the posterior LV free wall between the bases of the two PMs (Figure 3) has an effect on regional wall motion. Echocardiographic follow-up in our patients, who were all poor or borderline responders in terms of predictable remodeling, show that the LV diameters consistently shorten, ejection fraction increases, volumes melt, sphericity indexes improve, and the LV walls thicken, whereas functional status and survival are excellent. Acute surgical remodeling of the local LV anomalies that focus on the subvalvular mitral apparatus may therefore have long-term beneficial effect that will cumulate with the already consistent effect of mitral valvuloplasty.

\section{CONCLUSIONS}

Mitral repair by means of a sling encircling and reapproximating both PMs, setting a more normal " $\mathrm{PM}$-mitral annulus" anatomic relationship, completed by mitral annuloplasty seems a more rational approach in patients with ischemic MR. It restores mitral leaflet mobility, prevents recurrent MR, and shows an effect on ventricular dynamics in patients who are normally considered as poor responders to an undersized ring.

\section{References}

1. Yiu SF, Enriquez-Sarrano M, Tribouilloy C, Seward JB, Tajik AJ. Determinants of the degree of functional mitral regurgitation with systolic left ventricular dysfunction. A quantitive clinical study. Circulation. 2000;102:1400-6.

2. He S, Jiminez J, He Z, Yoganathan AP. Mitral leaflet geometry perturbations with papillary muscle displacement and annular dilatation: an in-vitro study of ischemic mitral regurgitation. J Heart Valve Dis. 2003;12:300-7.

3. Bolling SF, Pagani FD, Deeb GM, Bach DS. Intermediate term outcome of mitral reconstruction in cardiomyopathy. J Thorac Cardiovasc Surg. 1998;115:381-7.

4. Smolens IA, Pagani FD, Bolling SF. Mitral valve repair in heart failure. Eur J Heart Fail. 2000;2:365-71.

5. Tahta SA, Oury JH, Maxwell JM, Hiro SP, Duran CM. Outcome after mitral valve repair for functional ischemic mitral regurgitation. J Heart Valve Dis. 2002;11: $11-8$.
6. Matsunaga A, Tahta SA, Duran CM. Failure of reduction annuloplasty for functional mitral regurgitation. J Heart Valve Dis. 2004;13:390-7; discussion 397-8.

7. Raman J, Dixit A, Storer M, Hare D, Buxton BF. Geometric endo-ventricular patch repair of inferior left ventricular scars improves mitral regurgitation and clinical outcome. Ann Thorac Surg. 2001;72:S1055-8.

8. Wu AH, Aaronson KD, Bolling SF, Pagani FD, Welch K, Koelling TM. Impact of mitral valve annuloplasty on mortality risk in patients with mitral regurgitation and left ventricular dysfunction. J Am Coll Cardiol. 2005;45:381-7.

9. Hvass U, Tapia M, Baron F, Pouzet B, Abdelshafy M. Papillary muscle sling: a new functional approach to mitral repairs in patients with ischemic left ventricular dysfunction and functional mitral regurgitation. Ann Thorac Surg. 2003;75: 809-11.

10. Grigioni F, Enrique-Sarano M, Zehr KJ, Baily KR, Tajik AJ. Ischemic mitral regurgitation: long-term outcome and prognostic implications with quantitative Doppler assessment. Circulation. 2001;103:1759-64.

11. Sutton MG, Sharpe N. Left ventricular remodelling after acute myocardial infarction: pathophysiology and therapy. Circulation. 2000;105:2981-8.

12. Buckberg. GD, and the Restore Group. Ventricular shape and function in health and disease. Semin Thorac Cardiovasc Surg. 2001;13:504-13.

13. Braum J, Bax JJ, Versteegh M, Voigt PG, Holman ER, Klautz R, et al. Pre-operative left ventricular dimensions predict reversal remodelling following restrictive mitral annuloplasty in ischemic mitral regurgitation. Eur J Cardiovasc Surg. 2005;27:847-53.

14. Hung J, Guerrero JL, Handschumacher MD, Supple G, Sullivan S, Levine RA. Reverse ventricular remodelling reduces ischemic mitral regurgitation: echoguided device application in the beating heart. Circulation. 2002;106:2594-600.

15. Tibayan FA, Rodriguez F, Langer F, Zasio MK, Baily L, Liang D, et al. Annular or subvalvvular approach to chronic ischemic mitral regurgitation? J Thorac Cardiovasc Surg. 2005;129:1266-75.

16. Ramadan R, Al-Attar N, Mohammadi S, Ghostine S, Amzoun A, Therasse A, et al. Left ventricular infarct plication restores mitral function in chronic ischemic mitral regurgitation. J Thorac Cardiovasc Surg. 2005;129:440-2.

17. Kollar A, Kekesi V, Soos P, Juhasz-Nagy A. Left ventricular external plication: an indirect off-pump mitral annuloplasty method in a canine model. J Thorac Cardiovasc Surg. 2003;126:977-82.

18. Kron IL, Green GR, Cope JT. Surgical relocation of the posterior papillary muscle in chronic ischemic mitral regurgitation. Ann Thorac Surg. 2002;74:600-1.

19. Messas E, Guerrero JL, Handschumacher MD, Conrad C, Chow CM, Sullivan S, et al. Chordal cutting: a new therapeutic approach for ischemic mitral regurgitation. Circulation. 2001;104:1958-63.

20. Lomholt M, Nielson SL, Hansen SB, Anderson NT, Hasenkam JM. Differential tension between secondary and primary mitral chordae in an acute in-vivo porcine model. J Heart Valve Dis. 2002;11:337-45.

\section{Discussion}

Dr Robert A. Dion (Genk, Belgium). Dr Hvass, as you mentioned, our group in Leiden has demonstrated that a preoperative cutoff value of the LV end-diastolic diameter of $65 \mathrm{~mm}$ is an independent predictor of survival and of LV reverse remodeling. Below this value, a stringent undersizing annuloplasty by two sizes using a complete semirigid ring (Physio ring), as you also now use, yields a coaptation length of at least $8 \mathrm{~mm}$. In our hands, this is invariably efficient in correcting MR without producing mitral stenosis and also addresses LV remodeling, even after a follow-up of 5 years. We also concluded that something else should be added to the annuloplasty at the ventricular level in the presence of a more dilated left ventricle, in case the LV end-diastolic diameter is more than $65 \mathrm{~mm}$.

There are, in my opinion, two ways to address the ventricular level: either from the outside, such as using the CorCap, as we have chosen in Leiden with initial promising results, or from the inside, such as the PM resuspension described by Irving Kron and his group, or the PM approximation by stitches proposed by Matsui from Japan, or the PM sling that you already proposed a while ago. 
I have been convinced by this brilliant idea and have used it in addition to restrictive mitral annuloplasty in patients with dilated ventricles exceeding 65 -mm end-diastolic dimension with promising short-term results. Your patient cohort has a mean preoperative LV end-diastolic diameter of $70 \mathrm{~mm}$ and therefore indeed corresponds to the Leiden cohort, which had disappointing results: a mortality of $50 \%$ at 5 years and an absent or less significant reverse remodeling in only $50 \%$ of the survivors. Only 2 of 29 patients in your group died after 3 years and 2 others required a reoperation. This is a spectacular result, certainly if one considers the amount of continuous reverse remodeling that you described over 1 year.

I have a few questions. What is your current indication for performing a PM sling in this group? Do you perform it systematically or only in the presence of a dilated ventricle with more than $65-\mathrm{mm}$ end-diastolic diameter? Do you also take into account the inter-PM distance, as Matsui recommends, doing something above $3 \mathrm{~cm}$ ?

Dr Hvass. To answer the first question, I must admit that I do not see the good responders. I am essentially having referred to me the patients who already have very enlarged ventricles. It is more or less a systematic approach since I am not seeing the other patients, and I have been using it in all of these patients. It is true that in all of these patients, the distance of the PMs is much more than $3 \mathrm{~cm}$.

Dr Dion. Thank you. In the last 10 patients, you write in the manuscript that you no longer undersized the annuloplasty ring. Did you measure the length of coaptation in these patients in whom you did a sling with no restrictive mitral annuloplasty?

Dr Hvass. In the first group of patients, the ring was hardly undersized. I mean, it was not two sizes. I was using the size of the anterior leaflet as a guideline and sometimes wondering whether I should undersize it or not, just for safety. Then when I became very confident with the technique, I just took the normal size and have been continuing that way. You do not need to undersize mitral rings. When you have the mitral rings together, you have much more mobility of the leaflets.

The height of the coaptation is very good. I do not try to get 8 $\mathrm{mm}$ each time, as you advocate, but it is between 5 and $8 \mathrm{~mm}$ in all the cases. We never have very, very shallow coaptation heights with this technique.

Dr Dion. Thank you. In my limited experience with your technique, I ended up in a 5-year-old child after the sling with a prolapse of A1, A2, P1 after approximation of the PMs, probably because I approximated the PMs with different heights. Did you encounter this problem and have you considered additionally stitching together the tips of the PMs to prevent this problem and reinforce your intraventricular repair?

Dr Hvass. I remember you talked about this problem with me already, and I was a bit surprised because I have not encountered it in any of my patients. I do not know how I would deal with it. I do not know whether it would be necessary to put an additional stitch at the tip of the PMs or not.
Dr Steven F. Bolling (Ann Arbor, Mich). I disclose I have IP and royalty rights to the GeoForm ring.

Dr Hvass, congratulations on your pioneering work. This clearly is a ventricular problem and you have proposed a very interesting operation that perhaps is technically demanding for the rest of us. I have a particularly technical question. We know now that the closure cylinder of the mitral valve apparatus should be stacked as a cylinder. Do you measure the sling, do you make it the same size and diameter as the annuloplasty ring, or how do you size the sling?

Dr Hvass. I do not size it. I just put it around the PMs and bring the PMs into contact. That is the only measure I do. It has nothing to do with the size of the mitral ring above it. It may not be a complete cylinder, but that is the way I have been doing it.

Dr Richard J. Shemin (Los Angeles, Calif). No disclosures.

I have another question about intraoperative decision-making. Obviously, there is often variability in the sizes and the distribution of PMs, so what have you learned from your experience of when not to do this, and are PMs that are asymmetrical a problem in getting a good result?

Dr Hvass. As you saw from the slides, there was an initial mistake when I did leave a group of PMs out. I am very careful now to look at the exact anatomy, what is happening below, and go around all the PMs that are there.

Dr R. W. M. Frater (Bronx, $N Y$ ). Have you ever had to turn a patient down because of the irregularity of the anatomy?

Dr Hvass. No. I think it is quite unusual to have what is called a type 4 mitral PM anatomy. I think it is very unusual.

Dr David H. Adams (New York, NY). Dr Hvass, I would like to make a comment and then ask you a question. I think we have to stop presenting slides that say that current strategy fails in $20 \%$ to $50 \%$ of these patients. A lot of those patients were not downsized; a lot of those patients received partial flexible bands, which is not done in most centers. We should start talking about patients who had downsized remodeling rings and what that standard is from Leiden and others as the benchmark. There is a recurrence rate, probably $10 \%$ or $15 \%$, but I think it is historical now to say $50 \%$ of ischemic MR repairs fail. I think it sets the bar too low from the percutaneous side.

Having said that, I congratulate you for this really unique work in trying to move PMs, but I am going to ask you for some more science. Can you tell us about tethering angle, tethering area, and depth of coaptation? Your MR grades were grade 2 to 4 , so you can have a big ventricle and still have mild MR. I am impressed by your remodeling, but do you have more precise echo data on the amount of tethering and what you are doing to that after you do this operation compared with just annuloplasty?

Dr Hvass. We have data on the preoperative and postoperative tethering, which I have been showing in all the previous meetings. I decided not to show it this time because most people know it. We have had the preoperative tethering that is going from $16 \mathrm{~mm}$ to less than $4 \mathrm{~mm}$. There was a big, big change in that. 